\title{
Discovery of a PFKFB3 inhibitor for phase I trial testing that synergizes with the B-Raf inhibitor vemurafenib
}

\author{
Sucheta Telang ${ }^{1}$, Julie O'Neal', Gilles Tapolsky ${ }^{3}$, Brian Clem², Alan Kerr', Yoannis Imbert-Ferndandez', \\ Jason Chesney ${ }^{1 *}$
}

From Metabolism, Diet and Disease 2014: Cancer and metabolism

Washington DC, USA. 28-30 May 2014

\section{Background}

In human cancers, loss of PTEN, stabilization of hypoxia inducible factor- $1 \alpha$, and activation of Ras and AKT converge to increase the activity of a regulator of glycolysis, 6-phosphofructo-2-kinase/fructose-2,6-bisphosphatase (PFKFB3). This enzyme synthesizes fructose-2,6-bisphosphate (F2,6BP), which is an activator of 6-phosphofructo1-kinase, a key step of glycolysis that is tightly controlled by multiple metabolic feedback mechanisms. We recently identified the first competitive small molecule inhibitor of PFKFB3, 3-(3-pyridinyl)-1-(4-pyridinyl)-2-propen-1-one (3PO), and have now sought to develop a more potent PFKFB3 inhibitor with improved PK properties for testing in clinical trials.

\section{Materials and methods}

Methods included recombinant PFKFB3 assays, PK studies using mass spectrometry, pre-clinical toxicity and efficacy studies, Western blot analyses for HIF- $1 \alpha$ and PFKFB3 in A375 melanoma cells, F2,6BP assessments and flow cytometry for apoptosis.

\section{Results}

We report the discovery of a novel 3PO-derivative, PFK158, that is more potent than 3PO, has improved PK properties and causes $\sim 80 \%$ growth inhibition in several mouse models of human-derived tumors. We also demonstrate that PFK158 is well tolerated in rats and dogs providing key support for a phase 1 trial in cancer patients that will initiate in 2014. Once the MTD is established, we intend to conduct multiple phase $1 / 2$ trials of PFK158 in combination with targeted agents given the ability of PFK158 to suppress glycolysis. 50\% of melanomas harbor a $\mathrm{BRAF}^{\mathrm{V} 600 \mathrm{E}}$ mutation that promotes glucose metabolism, survival and proliferation and $\mathrm{BRAF}^{\mathrm{V} 600 \mathrm{E}}$ inhibitors are effective in $\sim 50 \%$ of melanoma patients. Unfortunately, resistance to these agents develops within six months and most patients die within two years of diagnosis. Genetic amplifications of $\mathrm{BRAF}^{\mathrm{V} 600 \mathrm{E}}$ are a common cause of resistance, and BRAF ${ }^{\mathrm{V} 600 \mathrm{E}}$ stabilizes HIF-1 $\alpha$, an established promoter of PFKFB3. We thus hypothesized that PFKFB3 may be essential for intrinsic resistance to $\mathrm{BRAF}^{\mathrm{V} 600 \mathrm{E}}$ inhibitors. In unpublished results, we demonstrate that 3 hours of exposure to $1 \mu \mathrm{M}$ vemurafenib reduces HIF-1 $\alpha$, PFKFB3, and F2,6BP in BRAF ${ }^{\mathrm{V} 600 \mathrm{E}}$ positive A375 melanoma cells and that PFK158 markedly sensitizes these cells to the apoptotic effects of vemurafenib.

\section{Conclusions}

In conclusion, PFK158 is the first PFKFB3 inhibitor to be examined in a phase I trial and may have significant clinical utility when combined with agents that target driver oncogenes. Importantly, our data provide rationale for the conduct of pre-clinical studies of PFK158 combined with vemurafenib in transgenic $\mathrm{BRAF}^{\mathrm{V} 600 \mathrm{E}}$ melanoma mouse models which are in turn expected to justify a phase $1 / 2$ trial of the combination in $\mathrm{BRAF}^{\mathrm{V} 600 \mathrm{E}}$-positive melanoma patients.

\footnotetext{
Authors' details

${ }^{1} \mathrm{JG}$ Brown Cancer Center, University of Louisville, Louisville, KY, USA. ${ }^{2}$ Department of Biochemistry, University of Louisville, Louisville, KY, USA. ${ }^{3}$ Advanced Cancer Therapeutics, Louisville, KY, USA.
} 
- Convenient online submission

- Thorough peer review

- No space constraints or color figure charges

- Immediate publication on acceptance

- Inclusion in PubMed, CAS, Scopus and Google Scholar

- Research which is freely available for redistribution 